\title{
Hardware Qualification Research On Digital Control System
}

\author{
Rui $\mathrm{Li}^{1 \text {, a }}$, Jianwen Qiu ${ }^{1, \mathrm{~b}}$ and Jishi Guan ${ }^{1, \mathrm{c}}$ \\ ${ }^{1}$ China Nuclear Power Technology Research Institute Beijing Devision,Beijing,100086,China \\ ali_rui@cgnpc.com.cn, bqiujianwen@cgnpc.com.cn, 'oguanjishi@cgnpc.com.cn
}

Keywords: Digital control system. Hardware qualification. Virtual instrument.

Abstract. As the acceleration of the process of localization, hardware qualification becomes more and more important for digital control system. This paper states the advantages of digital control system and importance of qualification. It summarizes the contents of qualification test. Based on virtual instrument, this paper develops a test system and proposes the hardware and software technical program for hardware qualification of digital control system.

\section{Introduction}

Digital control system, which is called nerve center of nuclear power plant and heart of master control room, is one of the four key equipments that can influence safety and stability of nuclear power plant.

For a long time, digital control system for nuclear power plant in China, which depends on foreign imports and lacks of independent technology, stays in low-end position in the industry. As rapid development of the nuclear power industry, localization of fully digital control system is one of the key factors which can constrain localization process in China. Digital control system has incomparable advantages than analog systems, which includes[1]:

1. Easy to realize advanced protection schemes and complex control algorithm.

2. Overcome the interference and drift effects and improve measurement accuracy.

3. Connected to the communication network to exchange information, greatly reducing the number of cables, improving the reliability of data transmission and reducing the maintenance workload.

4. Easily achieve redundancy, fail-safe and fault-tolerant based on fault-tolerant technology.

5. Convenient and effective online checking and self-diagnosis.

6. Open, standardized design and good flexibility and maintainability.

7. Friendly man-machine interface, reducing operator's misuse.

\section{Content of Hardware Qualification for Digital Control System}

Hardware qualification of digital control system is not only an important means to verify whether the hardware can meet the requirements of nuclear safety or not, but also the requirements of relevant laws and regulations.

Qualification has three stages: pre-test, qualification test and test after qualification.

1.Pre-test: System start-up and self-test, it includes hardware, software validation, and cable connections. Under standard conditions, it is also known as the reference test, which contains electrical characteristics test, such as dielectric withstand voltage test, insulation resistance measurement and so on.

2.Qualification test: it includes environmental test, long-term test, electromagnet-ic compatibility test, seismic test and so on.

3.Test after qualification: Running test, including resolution, precision of analog module, system response time, input and output of switching value, timer function, fault detection, fault diagnosis, power stability and so on. Validation test, including failure test of receiving port, port noise test, emergency test.

\section{Test System of Hardware Qualification for Digital Control System}

The system includes hardware and software. This part gives the test system of digital control system. 


\section{Digital Control System Block Diagram}

Digital control system is common platform of protection system in nuclear power plant[2], which can be used for safety class instrumentation and control systems in different types of nuclear reactor, including reactor protection system (RPS), post-accident monitoring system (PAMS), engineered safety features control system and so on. A typical system block diagram is shown as Fig.1.

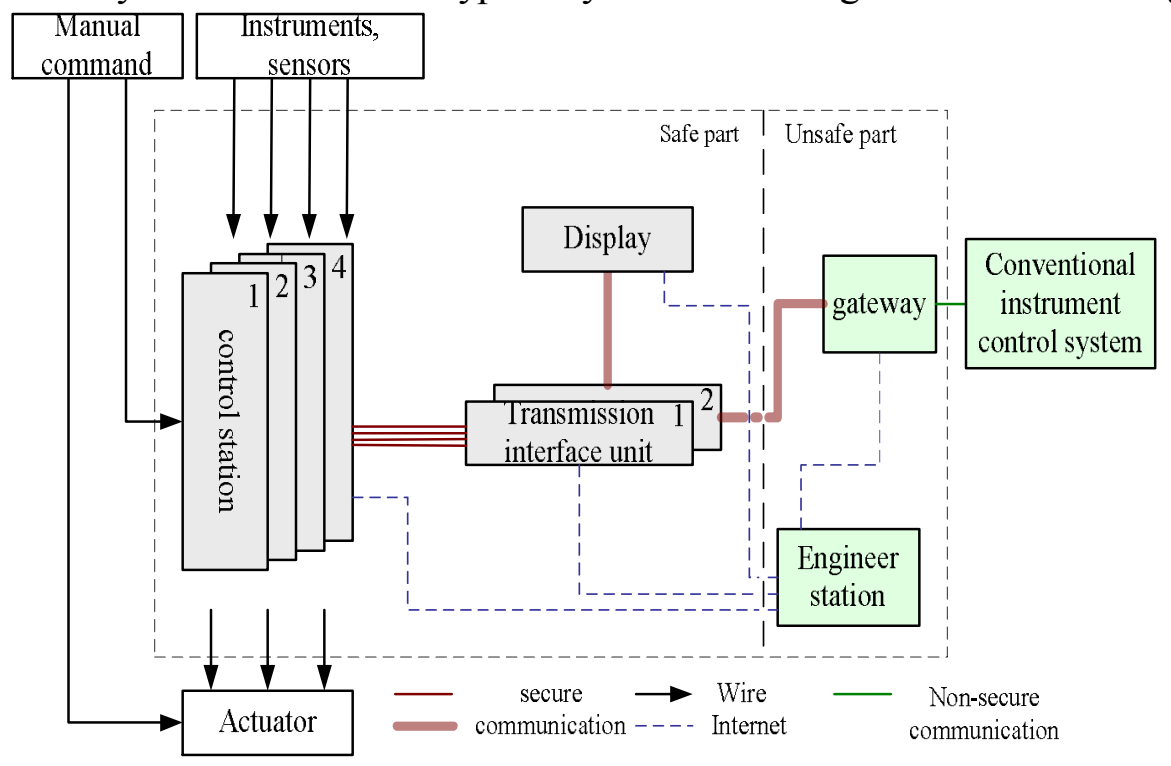

Fig. 1 Digital control system block diagram

\section{Hardware Design of Test System}

Test system can be designed based on PXI modular instrument architecture and virtual instrument technology, which is proposed by NI company. In order to achieve a single-channel or multi-channel input and output test, monitor response output and compare the data, a test system should be developed with input and output hardware interface devices and data processing system. A test system is shown as Fig.2.

The excitation and response device can be configured according to specimen and test requirements. In the test system, excitation signal device generates correct signals in the corresponding channel and on fixed time according to software instruction. Response signal device acquires signal of corresponding channel and transfers the data to PC for analysis. I/O interface device is the main body of test system, which looks like a bridge for data exchange and realizes logic level conversion and electrical connection between specimen and test system. Man-machine interface device composed by the display, mouse and keyboard, is responsible for receiving test instruction and sending data to system software. At the same time, it receives acquisition data to achieve real-time display.

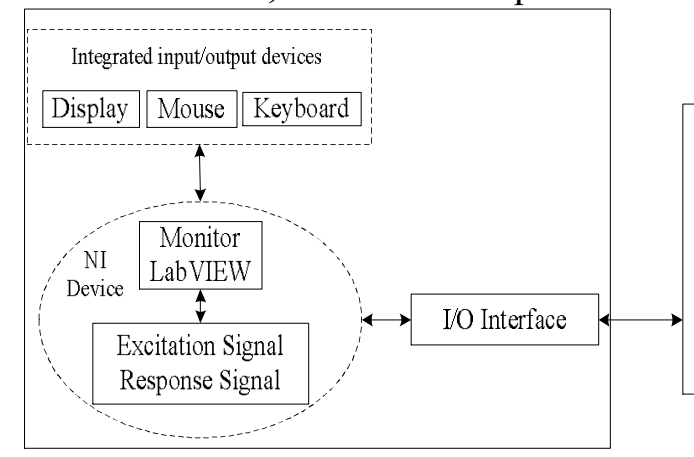

Test System

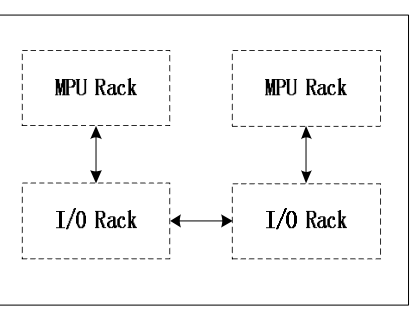

Specimen

Fig. 2 Test system block diagram 


\section{Software Design of Test System}

There are many tests and strict time requirement for hardware qualification for digital control system. Considering different objects, test contents and requirements change frequently, so software is designed based on modular design in or-der to ensure less modification after object changes. Part of the software program to ensure that changes occur for test object can be modified without affecting the entire software system. Specimen and test system are also digital systems using programmable controllers. Software has become key point of normal operation of the entire system.

Software is based on LabVIEW graphical programming environment which is virtual instrument technology. According to system requirement, software has been divided into five parts, which includes excitation module, response module, signal analysis module, data save module and report print module.

After test system starts up, according to the operator's instruction, test item should be selected from the program, which has stored in advance. And then excitation signal can be sent to specimen. Specimen can act and return response signal to test system. After test system received test data, it will implement data process and real-time display. According to the instruction, test system will judge if data save and report printing are needed or not. The detail process is shown as Fig.3.

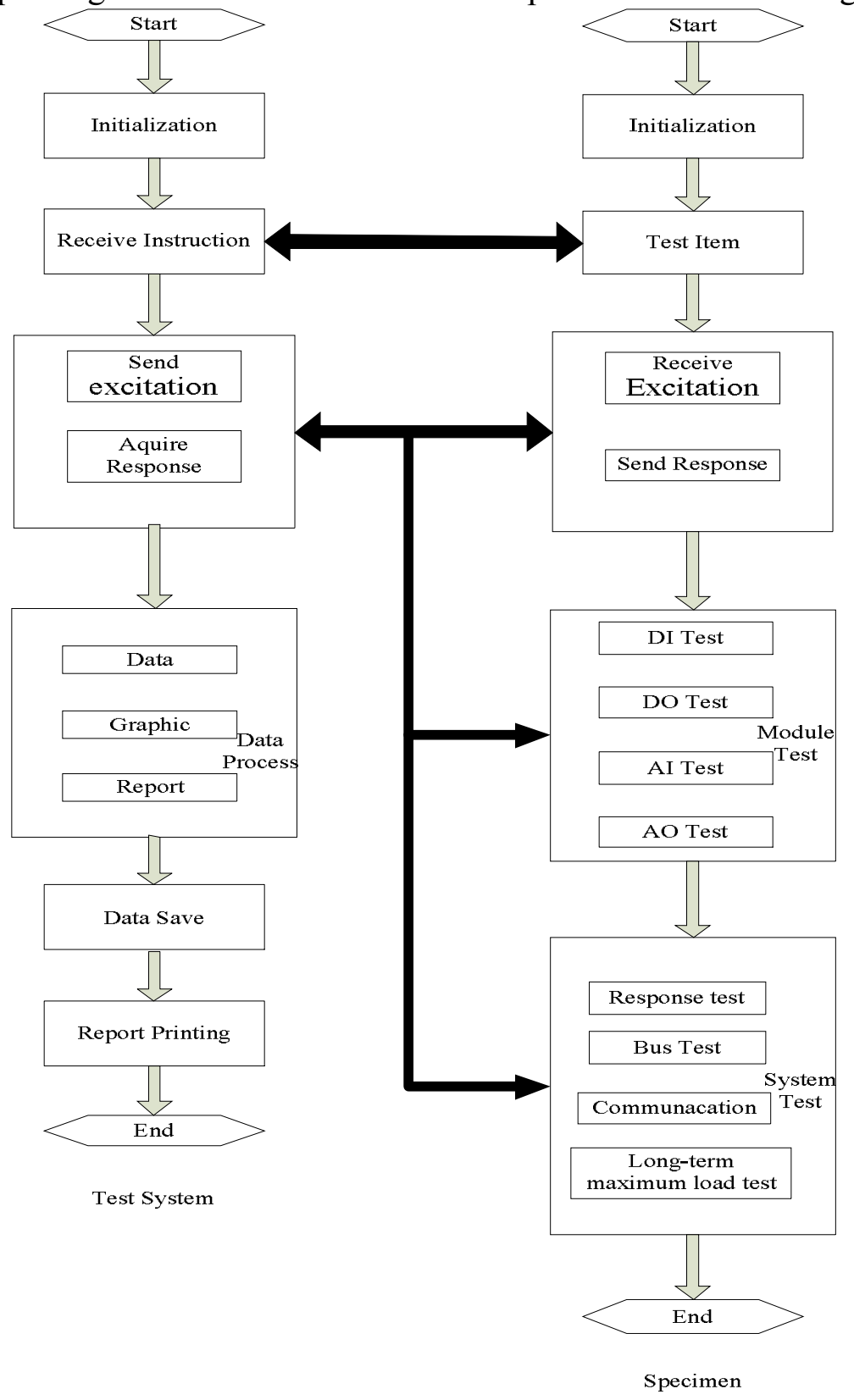

Fig. 3 Detail process of test flow 


\section{Conclusions}

As digital control system plays an important role in nuclear power plants, a complete hardware qualification techniques and methods should be established, so test procedures should be written and also developing a digital, net-worked, high precision and common test system become more and more useful. This paper discussed hardware qualification technology and proposed qualification content. This paper also proposed test system design based on virtual instrument technology .It will play a positive role in promoting hard-ware qualification for digital control system.

\section{References}

[1] Ji.Shi, Mingyu.Jiang, Jianchao.Zheng, Yunqing.Ma, Digital technology research status on nuclear power plant instrument and control (I\&C) system, Measurement and control technology, vol.2,pp29-32,,2004.

[2] Changli.Wang, An.Luo. Distributed control system (DCS) design and applications, Beijing: Electronic Industry Press. 